\title{
Impact of Early Infant Growth, Duration of Breastfeeding and Maternal Factors on Total Body Fat Mass and Visceral Fat at 3 and 6 Months of Age
}

\author{
Laura M. Breij ${ }^{a}$ Marieke Abrahamse-Berkeveld ${ }^{b}$ Dennis Acton ${ }^{b}$ \\ Emanuella De Lucia Rolfe ${ }^{c}$ Ken K. Ong ${ }^{c}$ Anita C.S. Hokken-Koelega ${ }^{a}$ \\ a Department of Pediatrics, Subdivision of Endocrinology, Erasmus MC/Sophia Children's Hospital, Rotterdam, The \\ Netherlands; ${ }^{b}$ Nutricia Research, Utrecht, The Netherlands; ${ }^{c}$ Medical Research Council Epidemiology Unit, University \\ of Cambridge, Institute of Metabolic Science, Cambridge Biomedical Campus, Cambridge, UK
}

\section{Keywords \\ Body composition · Fat mass · Visceral fat}

\begin{abstract}
Background: Accelerated gain in fat mass in the first months of life is considered to be a risk factor for adult diseases, given the tracking of infancy fat mass into adulthood. Our objective was to assess the influence of early growth, type of feeding and maternal variables on fat mass in early life. Methods: In 300 healthy term infants, we longitudinally measured fat mass percentage (FM\%) by air-displacementplethysmography at 1, 3, and 6 months and abdominal visceral and subcutaneous fat measured by ultrasound at 3 and 6 months. Results: Both gain in FM\% and weight-for-length in the first 3 months were positively associated with FM\% at 6 months of age and visceral fat at 3 months of age. Gain in FM\% and weight-for-length between 3 and 6 months were both positively associated with visceral fat at 6 months. Breastfeeding duration associated positively with subcutaneous fat but not with visceral fat at 3 and 6 months. Mater-
\end{abstract}

nal characteristics did not associate with FM\% or visceral fat at 3 or 6 months. Conclusion: Higher gain in FM\% or in weight-for-length in the first postnatal months leads not only to higher FM\% but also more to accumulation of visceral fat. Exclusive breastfeeding appears to promote subcutaneous but not visceral fat in the first 6 months.

(c) 2017 S. Karger AG, Basel

\section{Introduction}

The first 3 months of life are known to be a critical window for the programming of adiposity and cardiovascular diseases [1-3]. Unraveling the modifiable determinants that influence the adiposity and fat mass development in early life can provide valuable insights to support an optimal infant development.

We have previously shown that the risk for type 2 diabetes and cardiovascular diseases at the age of 21 years differs based on weight-for-length gain during the first months of life, indicating that early growth might be a de-

\section{KARGER}

(c) 2017 S. Karger AG, Basel

E-Mail karger@karger.com

www.karger.com/anm

\footnotetext{
Laura M. Breij

Erasmus University Medical Center/Sophia Children's Hospital

Room number: Sk-0152, Wytemaweg 80

NL-3015 CN Rotterdam (The Netherlands)

E-Maill.breij@erasmusmc.nl
} 
terminant for later life metabolic health [4]. One of the key elements driving early life growth is the nutrition provided to the young infant. Exclusive breastfeeding is the preferred method of feeding for newborn infants, this method being associated with a.o. less infections, better cognitive development, but also with a lower incidence of childhood obesity and types 1 and 2 diabetes [5]. However, outcomes of studies on the influence of breastfeeding on infant's body composition are not straightforward [6-9].

Apart from postnatal factors, a higher pre-pregnancy maternal weight and maternal weight gain during pregnancy have been associated with a higher infant weight and fat mass percentage (FM\%) at birth $[10,11]$. Given the increasing prevalence of overweight in adult women, including those at reproductive ages [12], this could potentially contribute to a cross-generational vicious obesity circle. However, it is not known whether these maternal factors have a lasting influence on infant adiposity development. Most studies have focused on weight, length, and other anthropometric measures as a proxy for adiposity in infancy, instead of accurate body composition, but with air-displacement plethysmography (ADP), infants' body composition (i.e., fat and fat-free mass) can be measured accurately [13-15].

Total body fat has adverse consequences on adult disease risks, but location of body fat seems to be even more important. Abdominal visceral fat mass during childhood is associated with an unfavorable metabolic profile in later life [16, 17]. Recently, an ultrasound methodology has been validated to measure infant visceral fat and abdominal subcutaneous fat, enabling a noninvasive approach to obtain more insights into the development of these fat depots during infancy [18].

Our aim was to identify the determinants of total FM\%, as well as visceral and abdominal subcutaneous fat in the first months of life. Our primary hypothesis was that, independent of birth weight, early weight gain between birth and 3 months and more specifically the gain in FM\% between 1 and 3 months of age are associated with a higher fat mass and visceral fat mass at 3 and 6 months. Our secondary hypothesis was that longer duration of exclusive breastfeeding is associated with more subcutaneous fat. Our third hypothesis was that prepregnancy BMI and maternal weight gain during pregnancy are associated with a higher infant FM\% and visceral fat mass at 3 and 6 months.

We, therefore, measured in a cohort of healthy term infants' longitudinal total body composition by ADP at 1 ,
3 , and 6 months of age as well as visceral and abdominal subcutaneous fat by ultrasound at 3 and 6 months of age and associated these outcomes with infant data, type of feeding and maternal variables.

\section{Material and Methods}

\section{Subjects}

The study population consisted of 300 healthy term infants, who are embedded in a larger birth cohort study (Sophia Pluto Study) aimed at examining the postnatal determinants of body composition during infancy. Infants were recruited from several hospitals in and near Rotterdam, a large city in the Netherlands (600,000 habitants). All participants fulfilled the same inclusion criteria: (1) born at term ( $\geq 37$ weeks of gestation), (2) age at recruitment $<28$ days, and (3) uncomplicated neonatal period without severe asphyxia (defined as an Apgar score below 3 after $5 \mathrm{~min}$ ), sepsis or long-term complication of respiratory ventilation. Exclusion criteria were known congenital or postnatal diseases that could interfere with body composition development, confirmed intra-uterine infection, maternal use of corticosteroids or a maternal medical condition that could interfere with infant's body composition development (e.g., diabetes). The Medical Ethics Committee of Erasmus Medical Center approved the study. Written informed consent was obtained from both parents unless the mother was single.

\section{Data Collection and Measurements}

Parental and Pregnancy Characteristics

Maternal data, that is, pre-pregnancy weight and highest weight in pregnancy, height, parity, smoking, ethnicity and complications during pregnancy, was obtained from medical records and questionnaires.

The Institute of Medicine published in 2009 revised gestational weight gain guidelines to minimize the negative health consequences for both mother and foetus of inadequate or excessive gain. They include 4 classifications of preconception body mass index (BMI; World Health Organization definitions); underweight, normal weight, overweight and obese. Maternal underweight was defined as a pre-pregnancy BMI $<18.5 \mathrm{~kg} / \mathrm{m}^{2}$, overweight as a $B M I \geq 25 \mathrm{~kg} / \mathrm{m}^{2}$, and obesity as a BMI $\geq 30 \mathrm{~kg} / \mathrm{m}^{2}$. Weight gain recommendations that were given per pre-pregnancy BMI-category include 3 categories, too less weight gain, normal weight gain and too much weight gain [19].

Information regarding socioeconomic status and educational levels of parents were obtained using questionnaires.

The Dutch Standard Classification of Education was used to categorize mothers to one of 4 levels of education: high (university degree), mid-high (higher vocational training, Bachelor's degree), mid-low ( $>3$ years general secondary school, intermediate vocational training), low (no education, primary school, lower vocational training or 3 years or less general secondary school) [20].

Infant Characteristics

Research clinic visits were scheduled at ages 1,3, and 6 months. Birth data, such as gestational age, was collected from midwife and hospital records. Information on breast and formula feeding was asked at the clinic visits. 
Anthropometrics: weight was measured to the nearest gram by an electronic infant scale (Seca, Hanover, MD, USA); length was measured twice in all infants by the same 2 persons using the 2 -persons technique to the nearest $0.1 \mathrm{~cm}$ by a length measuring board (Seca). In case of $>5 \mathrm{~mm}$ deviation between the 2 measurements, an additional measurement was performed and the mean of the measurements closest together were used. SD scores for birth length, birth weight, weight, length and weight for length were calculated to correct for gestational age and gender with Growth Analyser Research Calculation Tools 4.0 (available at www. growthanalyser.org), according to Dutch age- and gender-matched reference values [21].

Body composition: whole-body composition was estimated using ADP using the Peapod, Infant Body Composition System (COSMED) $[14,15,22,23]$. Briefly, this ADP system assesses fat mass, FM\% and fat-free mass and fat-free mass percentage by direct measurements of body volume and body mass, based on the whole-body densitometric principle. All measurements were obtained by experienced personnel, according to standardized protocol. The Peapod was calibrated every day, according to the protocol recommended by the supplier.

Abdominal fat: visceral and abdominal subcutaneous fat were estimated at 3 and 6 months using a Prosound 2 ultrasound (US), with a UST-9137 convex ultrasound transducer (both from Hitachi Aloka Medical, Zug, Switzerland). Two experienced researchers performed all US measurements after an extra training to measure subcutaneous and visceral fat in infants. To assess the intra-observer and inter-observer repeatability of the measurements, we calculated the intra-class and inter-class correlation coefficients. The inter-class correlation coefficients ranged from 0.75 to 0.97 , indicating that our measurements were reproducible. For both measures, the transducer was positioned where the xiphoid line intercepted the waist circumference measurement plane. Visceral fat was estimated by measuring the visceral depth, which is the distance measured in centimeters between the peritoneal boundary and the corpus of the lumbar vertebra, assessed in the longitudinal plane with the ultrasound probe depth set at $9 \mathrm{~cm}$. Subcutaneous abdominal fat was estimated by the distance in $\mathrm{cm}$ between the cutaneous boundary and the linea alba at the same location but on a transverse plane with a probe depth of $4 \mathrm{~cm} \mathrm{[18].}$

\section{Statistical Analysis}

The number of infants at birth and at 1,3 , and 6 months is shown in online supplemental Figure 1 (see www.karger.com/ doi/10.1159/000481539). Baseline characteristics are expressed as means and SD because variables were normally distributed. Linear regression analyses were performed to determine associations between birth weight SDS, birth length SDS, change in body FM\% (delta FM\% ${ }_{1-3 \text { mo }}$ ) between 1 and 3 months of age, duration of exclusive breastfeeding, maternal pre-pregnancy BMI (as exact value and as category according to [19]), maternal weight gain during pregnancy (as exact value and as category per pre-pregnancy BMI [19]), and the outcome variables: FM\%, visceral and abdominal subcutaneous fat at the age of 3 and 6 months, with adjustment for gender and age.

Multiple linear regression (MR) analysis was performed to determine the variables that contributed to the FM\% and visceral and abdominal subcutaneous fat at the age of 6 months. All models were adjusted for birth weight SDS. First, we entered age, gender, duration of exclusive breastfeeding to the model (model A). Second, we added the change in FM\% from age 1 to 3 months (delta FM\% ${ }_{1-3 \text { mo; }}$ model B). Third, we entered the change in weight for length SDS from age 0 to 1 months (delta W/LSDS ${ }_{0-1 \mathrm{mo}}$ ) instead of the delta FM\% ${ }_{1-3 m o}$ (model C). Finally, the change in weight for length SDS from age 1 to 3 months (delta W/LSDS $1-3$ mo $)$ was added instead of delta W/LSDS ${ }_{0-1 \text { mo }}$ (model D).

SPSS statistical package version 20.0 (SPSS Inc., Chicago, IL, USA) was used for analysis. Results were performed 2-sided and were regarded statistically significant if $p$ was $<0.05$.

\section{Results}

\section{Clinical Characteristics}

Maternal and infant demographic characteristics are presented in Table 1, including the numbers of infants at every visit. The mean age of the mothers was 32.3 years and gestational age was 39.7 weeks. Fifty-eight percent of the infants were male and $87 \%$ were Caucasian.

Mean FM\% increased from $16.7 \%$ at 1 month to $22.8 \%$ at 3 months and $23.8 \%$ at 6 months of age, whereas mean visceral fat remained stable between 3 and 6 months of age. At the start of the study, 240 infants received exclusive breastfeeding (80\%), at 1 month 165 infants (55\%), at 3 months 114 infants (38\%) received exclusive breastfeeding and at 6 months 57 infants received exclusive breastfeeding. The mean (SD) duration of breastfeeding was 16 (12) weeks.

Mean (SD) maternal pre-pregnancy BMI was 24.5 (4.7) $\mathrm{kg} / \mathrm{m}^{2}$ and maternal weight gain during pregnancy was 14.0 (10.4) kg. Of all mothers, 4\% had an underweight pre-pregnancy BMI, $61 \%$ had a normal pre-pregnancy BMI, 22\% an overweight pre-pregnancy BMI and 13\% an obese pre-pregnancy BMI.

Weight gain during pregnancy differed between maternal pre-pregnancy BMI-category $(p<0.001)$ with a substantially lower weight gain in obese mothers.

\section{Linear Correlations with Infant FM\% at 6 Months}

Table 2 shows the linear correlations of infant and maternal variables with FM\% at 3 and 6 months, corrected for gender and age.

Gain in FM\% (delta FM\%1-3mo) and in weight for length SDS (delta W/LSDS ${ }_{1-3 \text { mo }}$ ) between 1 and 3 months of life were both positively associated with FM\% at 6 months (all $p<0.001$ ). The duration of exclusive breastfeeding was positively associated with FM\% at 6 months $(p<0.01)$. None of the selected maternal variables including pre-pregnancy BMI and maternal weight gain during 
Table 1. Maternal and infant characteristics

Mean SD

\begin{tabular}{lcc} 
Infant characteristics & & \\
Gender, boys, \% & 58 & \\
Mode of delivery (cesarean delivery), \% & 32.3 & \\
Gestational age, weeks & 39.7 & 1.2 \\
Birth weight SDS & -0.38 & 1.1 \\
Birth length SDS & -0.23 & 1.2 \\
At 1 month ( $n=300)$ & & \\
Weight SDS & -0.30 & 1.2 \\
Length SDS & 0.03 & 0.9 \\
FM\% & 16.7 & 4.6 \\
At 3 months ( $n=268$ ) & & \\
Weight SDS & 0.47 & 1.1 \\
Length SDS & 0.36 & 0.9 \\
FM\% & 22.8 & 5.1 \\
US-visceral fat, cm & 2.54 & 0.6 \\
US-abdominal subcutaneous fat, cm & 0.42 & 0.1 \\
At 6 months $(n=248)$ & & \\
$\quad$ Weight SDS & 0.20 & 1.0 \\
Length SDS & 0.23 & 0.8 \\
FM\% & 23.8 & 5.3 \\
US-visceral fat, cm & 2.47 & 0.6 \\
US-abdominal subcutaneous fat, cm & 0.44 & 0.1 \\
Duration of breastfeeding (weeks) & 16 & 12 \\
Exclusive breastfeeding at 1st month, \% & 55 & \\
Exclusive breastfeeding at 3rd month, \% & 38 & \\
Exclusive breastfeeding at 3rd month, \% & 19 & \\
\hline
\end{tabular}

\section{Maternal characteristics}

Age, years

Height, cm

Pre-pregnancy weight, kg

Pre-pregnancy BMI, $\mathrm{kg} / \mathrm{m}^{2}$

Weight gain during pregnancy, $\mathrm{kg}$

Highest weight in pregnancy, $\mathrm{kg}$

Highest BMI in pregnancy, $\mathrm{kg} / \mathrm{m}^{2}$

Weight gain per pre-pregnancy BMI-category*

Underweight pre-pregnancy BMI $(<18.5)$ (4\%)

Normal pre-pregnancy BMI (18.5-24.9) (61\%)

Overweight pre-pregnancy BMI (25.0-30.0) (22\%)

Obese pre-pregnancy BMI (>30.0) (13\%)

Smoking during pregnancy, $\%$

Caucasian ethnicity, \%

Educational level, \%

High

Mid-high

Mid-low

$32.3 \quad 4.8$

168

$69.7 \quad 13.6$

$24.5 \quad 4.7$

$14.0 \quad 10.4$

$83.8 \quad 16.1$

$29.4 \quad 5.6$

15.7

4.9

$14.1 \quad 8.5$

$\begin{array}{ll}17.4 & 7.8\end{array}$

$\begin{array}{ll}7.3 & 18.5\end{array}$

4.7

87

26

25

21

Low

Other or unknown

* According to the 2009 IOM guidelines [19]. pregnancy were associated with FM\% at 6 months. Similarly, there was no relation between the different prepregnancy BMI categories or weight gain categories per pre-pregnancy BMI and the FM\% at 6 months.

\section{Associations with Visceral Fat at 3 and 6 Months}

Table 3 shows the linear associations, corrected for gender and age, of infant and maternal variables with visceral and subcutaneous fat at 3 and 6 months.

\section{Visceral Fat}

Delta FM\% ${ }_{1-3 \text { mo }}$ was positively associated with visceral fat at 3 months $(p=0.02)$ and delta FM\% $\%_{3-6 m o}$ with visceral fat at 6 months $(p=0.02)$. Delta FM\% ${ }_{1-3 \text { mo }}$ was not associated with visceral fat at 6 months $(p=0.99)$. Similarly, delta W/LSDS ${ }_{1-3 m o}$ was positively associated with visceral fat at 3 months $(p=0.01)$, but not at 6 months $(p=0.95)$, while the delta W/LSDS $3-6$ mo showed a positive trend with visceral fat at 6 months $(p=0.06)$.

\section{Subcutaneous Fat}

Delta FM\% ${ }_{1-3 m o}$ was positively associated with subcutaneous fat at 3 and 6 months $(p=0.01, p<0.001$, respectively) and delta $\mathrm{FM} \%_{3-6 \mathrm{mo}}$ with subcutaneous fat at 6 months $(p<0.001)$. Delta W/LSDS ${ }_{1-3 \text { mo }}$ was not associated with subcutaneous fat at 3 months $(p=0.16)$, but both delta W/LSDS ${ }_{1-3 \mathrm{mo}}$ and delta W/LSDS $3-6$ mo were associated with subcutaneous fat at 6 months (both $p<$ 0.001 ).

The duration of exclusive breastfeeding was positively associated with subcutaneous fat at 3 and 6 months $(p=$ $0.01, p=0.01$, respectively) but not with visceral fat (Table 3$)$.

Maternal pre-pregnancy BMI was associated neither with infant visceral nor with subcutaneous fat at 3 and 6 months (neither as exact increase nor based on category). Also weight gain during pregnancy was not associated with visceral or subcutaneous fat at 3 and 6 months (neither as exact increase nor based on category). Parity was only positively associated with subcutaneous fat at 6 months $(p=0.02)$. Other maternal variables were not associated with visceral or subcutaneous fat.

\section{Determinants of FM\%, Visceral and Subcutaneous Fat} at 6 Months of Age

To identify the variables that were the most important determinants of FM\% at 6 months, we performed MR analyses (Table 4). Model A shows that female gender and duration of exclusive breastfeeding were positively associated with $\mathrm{FM} \%$ at 6 months $(\beta=2.719, p<0.001$ 
Table 2. Associations of FM\% at the 3rd and 6 th months with infant and maternal variables

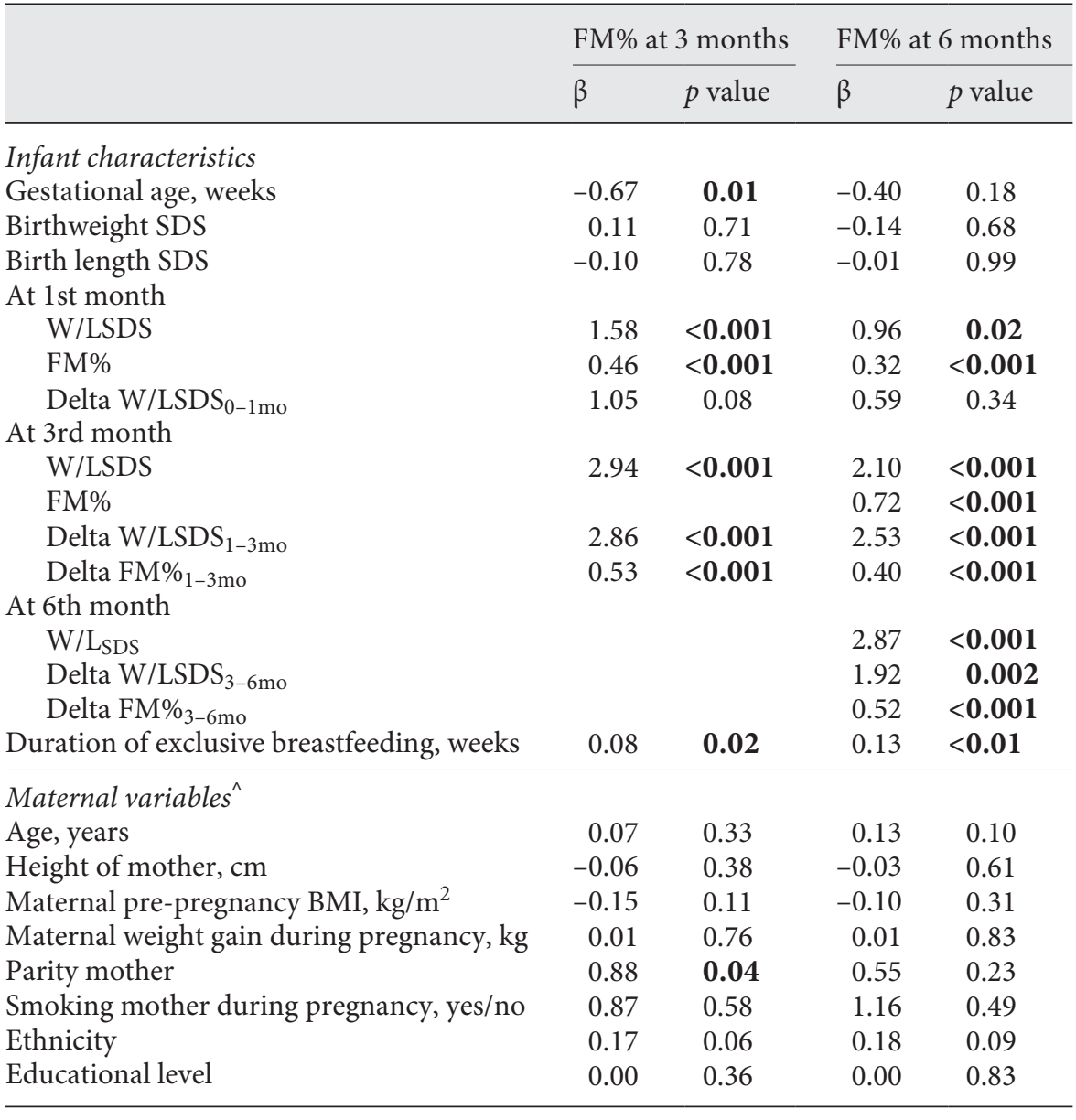

Values presented are results of multiple linear regression. $\beta$, unstandardized regression coefficient. All models are adjusted for gender and age.

${ }^{\wedge}$ Adjusted for birth weight. W/L $\mathrm{L}_{\mathrm{SDS}}$, weight for length SDS. Delta W/L $\mathrm{SDS}$, gain in weight for length SDS. Delta FM\%, gain in FM\%. Significant $p$ values are indicated in boldface. and $\beta=0.130, p<0.001$, respectively). The change in FM\% between the age of 1 and 3 months (delta FM\% ${ }_{1-3 m o}$ ) was positively associated with FM\% at 6 months next to female gender and duration of exclusive breastfeeding (model B). The change in W/L SDS between birth and 1 month (delta W/LSDS ${ }_{0-1 \mathrm{mo}}$ ) was not associated (model C), but the change in W/L SDS between 1 and 3 months (delta W/LSDS ${ }_{1-3 \mathrm{mo}}$ ) was positively associated with FM\% at 6 months $(\beta=2.249, p<0.001)$, next to female gender and duration of exclusive breastfeeding (model D).

As the gain in FM\% (delta FM\% ${ }_{3-6 \mathrm{mo}}$ ) and gain in weight for length delta W/LSDS $3-6$ mo $)$ between 3 and 6 months (delta FM\% $\%_{3-6 \mathrm{mo}}$ ) were the only determinants of visceral fat at 6 months, we did not investigate MR models for 6 month of age.

Determinants of Body Composition in Early Life

\section{Discussion}

In this longitudinal study with detailed body composition data during infancy, we found a strong association of gain in $\mathrm{FM} \%$ and its proxy weight-for-length SDS in the first 3 months of life with FM\% at 6 months. Gain in FM\% and in weight-for-length SDS between 1 and 3 months was associated with visceral fat at 3 months and a higher gain in FM\% between 3 and 6 months was associated with more visceral fat at 6 months. Exclusive breastfeeding duration was positively associated with FM\% and subcutaneous fat at 6 months but not with visceral fat.

The present study shows that especially the gain in FM\% between 1 and 3 months leads to a higher FM\% 
Table 3. Associations of ultrasound measurements at the $3 \mathrm{rd}$ and 6 th months with infant and maternal variables

\begin{tabular}{|c|c|c|c|c|c|c|c|c|}
\hline & \multicolumn{4}{|c|}{ At 3 months } & \multicolumn{4}{|c|}{ At 6 months } \\
\hline & $\beta$ & $p$ value & $\beta$ & $p$ value & $\beta$ & $p$ value & $\beta$ & $p$ value \\
\hline \multicolumn{9}{|l|}{ Infant variables } \\
\hline Gestational age, weeks & -0.05 & 0.13 & 0.01 & 0.07 & 0.00 & 0.74 & 0.00 & 0.68 \\
\hline Birthweight SDS & -0.02 & 0.67 & 0.00 & 0.35 & 0.04 & 0.83 & 0.00 & 0.84 \\
\hline \multicolumn{9}{|l|}{ At 1 st month } \\
\hline W/LSDS & 0.02 & 0.76 & 0.02 & 0.04 & 0.03 & 0.52 & 0.02 & 0.05 \\
\hline FM\% & -0.01 & 0.11 & 0.01 & 0.01 & 0.00 & 0.62 & 0.00 & 0.30 \\
\hline Delta W/LSDS ${ }_{0-1 \mathrm{mo}}$ & -0.13 & 0.07 & 0.05 & 0.002 & 0.07 & 0.31 & 0.00 & 0.88 \\
\hline \multicolumn{9}{|l|}{ At 3 rd month } \\
\hline $\mathrm{W} / \mathrm{L}_{\mathrm{SDS}}$ & 0.07 & 0.20 & 0.04 & $<0.001$ & 0.04 & 0.44 & 0.04 & $<0.001$ \\
\hline W/LSDS & & & & & 0.10 & 0.02 & 0.05 & $<0.001$ \\
\hline FM\% & & & & & 0.09 & 0.09 & 0.01 & $<0.001$ \\
\hline Delta W/LSDS $3-6$ mo & & & & & 0.14 & 0.06 & 0.04 & 0.004 \\
\hline Delta FM\% ${ }_{3-6 \mathrm{mo}}$ & & & & & 0.03 & 0.02 & 0.01 & $<0.001$ \\
\hline Duration of exclusive breastfeeding, weeks & 0.00 & 0.49 & 0.00 & 0.01 & 0.00 & 0.74 & 0.00 & 0.01 \\
\hline \multicolumn{9}{|l|}{ Maternal variables } \\
\hline Age, years & 0.00 & 0.91 & 0.00 & 0.57 & 0.02 & 0.13 & 0.00 & 0.06 \\
\hline Height of mother, $\mathrm{cm}$ & -0.01 & 0.08 & 0.00 & 0.64 & -0.01 & 0.19 & 0.00 & 0.05 \\
\hline Maternal pre-pregnancy BMI, $\mathrm{kg} / \mathrm{m}^{2}$ & 0.01 & 0.43 & 0.00 & 0.13 & 0.00 & 0.18 & 0.00 & 0.57 \\
\hline Maternal weight gain during pregnancy, kg & 0.01 & 0.14 & 0.00 & 0.46 & 0.00 & 0.18 & 0.00 & 0.20 \\
\hline Parity mother & -0.02 & 0.79 & 0.02 & 0.15 & 0.07 & 0.17 & 0.02 & 0.02 \\
\hline
\end{tabular}

Values presented are results of multiple linear regression. $\beta$, unstandardized regression coefficient. All models are adjusted for gender and age. $\mathrm{W} / \mathrm{L}_{\mathrm{SDS}}$, weight for length SDS; Delta $\mathrm{W} / \mathrm{L}_{\mathrm{SDS}}$, gain in weight for length SDS; delta FM\%, gain in FM\%.

Significant $p$ values are indicated in boldface.

Table 4. Multiple regression for FM\% at the 6th month

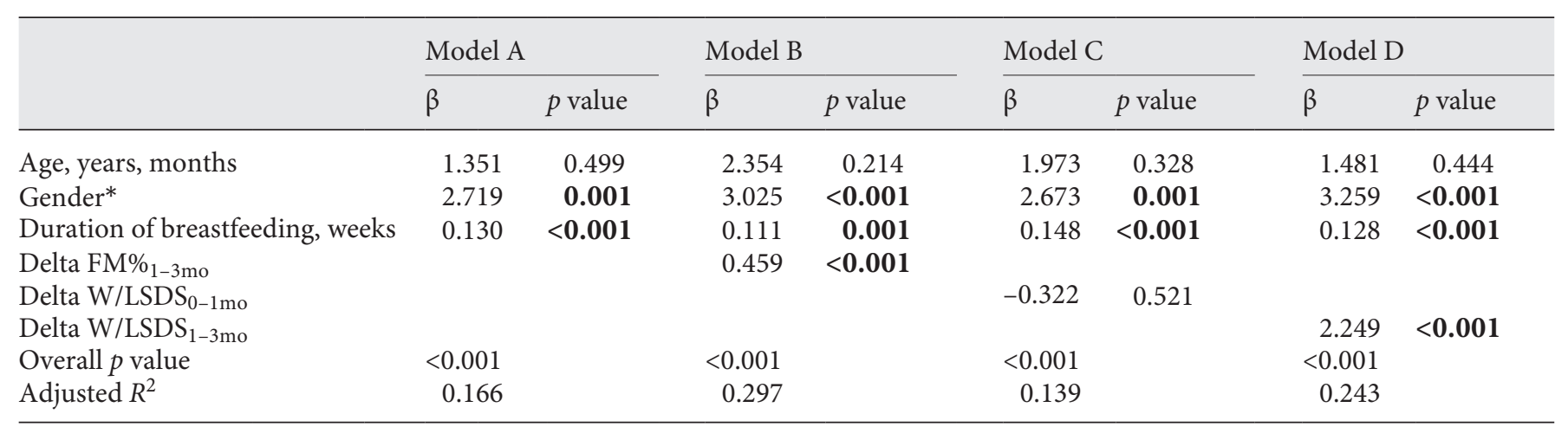

* Gender: 0, boys; 1, girls; W/L $\mathrm{L}_{\mathrm{SDS}}$, weight for length SDS; Delta W/L $\mathrm{L}_{\mathrm{SDS}}$, gain in weight for length SDS; Delta FM\%, gain in FM\%; Significant $p$ values are indicated in boldface. All models were adjusted for birth weight SDS. 
at 6 months and more visceral fat at 3 months. We have previously shown that infants with a higher FM\% at 6 months, measured by DXA scan, tended to keep this higher FM\% during childhood [24] and other studies showed that visceral fat in early life tends to track into childhood and adulthood [25-27]. Our results show that this might start as early as the first 3 months of life, emphasizing the need to monitor growth of infants closely from birth onwards. Longterm follow-up of our study will hopefully reveal the potential long-term effects of the early growth trajectory on the amount and location of adiposity in this population.

To our knowledge, this is the first study investigating the influence of predefined determinants of infant FM\% as well as visceral and subcutaneous fat during the first 6 months of life. Infant growth velocity in the first 3 months of life, represented by gain in weight-forlength SDS was associated with higher FM\% at 6 months. The impact of infancy weight for length SDS on body fat $\%$ might mediate its previously reported association with type 2 diabetes and cardiovascular diseases risk [28]. Moreover, these data support our previous findings, showing that young adults at the age of 21 years had higher risk for type 2 diabetes and cardiovascular diseases when they had a higher gain in weight for length SDS in the first 3 months, while after 3 months no associations between adiposity and risk factors at 21 years could be found. Our findings are in line with the results of previous reports $[2,29]$.

We found no association between total body FM\% and visceral fat, which is in line with the finding that in children, total body FM\% is more associated with subcutaneous fat rather than with visceral fat [30]. Our data show that associations with total FM\% cannot be simply extrapolated to visceral fat and underline the need for detailed body composition assessment instead.

In contrast to our expectations, infants who were still exclusively breastfed at 6 months had a higher FM\% at 6 months than those being exclusive formula fed. Exclusive breastfeeding during infancy has been associated with a lower risk of childhood obesity [31], but apparently this might not be caused by tracking of FM\% from infancy. Interestingly, we found that the higher FM\% associated with increased breastfeeding duration, could be primarily explained due to more subcutaneous fat and not to more visceral fat. One could postulate that if this difference in body fat distribution lasts throughout childhood, breastfed infants might have a more beneficial adi- posity phenotype with a reduced risk for obesity and adult diseases [32].

Apart from the type of milk feeding, other parental and heritable factors might have more impact on the body composition and obesity risk of their infants. Surprisingly, maternal variables, like pre-pregnancy BMI, were not associated with infant FM\% at 3 and 6 months. In a previous study, we showed that maternal BMI before pregnancy associates with FM\% at birth [33] and also other studies have shown associations between maternal variables and FM\% of newborns [10, 34], but apparently this is a transient effect that disappears after the first month of life. Our findings are in line with another study, showing that maternal BMI and weight gain during pregnancy had no influence on $\mathrm{FM} \%$, measured by DXA, in infants at 6 months of age [35]. In addition, the current study demonstrates that the association between maternal variables and FM\% of the infants as present at birth disappeared at the age of 3 months.

In conclusion, our study shows that a higher gain in FM\% in the first 3 months of life leads to more FM\% at 6 months of age and more visceral fat at 3 months. Similar associations were found with a higher gain in weight for length during the first months of life. These might underlie the reported long-term associations between rapid changes in weight for length SDS in early life and risks for obesity and adult diseases in later life. We also show that the consistently reported association between exclusive breastfeeding and higher total body FM\% in mid-infancy appears to be due to higher subcutaneous fat but not visceral fat.

\section{Acknowledgements}

We thank all the patients and their parents for participating in this study. We greatly acknowledge Mrs. J. van Nieuwkasteele and M. Huibregtse-Schouten, research nurses, for their assistance with the data collection. This study was an investigator-initiated study. A.C.S.H.-K. received an independent research grant by Nutricia Research. The concept of the study was developed by A.C.S.H.-K. and L.M.B. Data analysis and drafting the manuscript were primarily done by L.M.B. and A.C.S.H.-K., M.A.-B., D.A., E.D.L.R., and K.K.O. participated in data interpretation. All authors were involved in writing the manuscript and had final approval of the submitted version.

\section{Disclosure Statement}

M.A.-B. and D.A. are employees of Nutricia Research. This study was an investigator-initiated study. A.C.S.H.-K. received an independent research grant by Nutricia Research. 


\section{References}

$>1$ Breij LM, Kerkhof GF, Hokken-Koelega AC: Accelerated infant weight gain and risk for nonalcoholic fatty liver disease in early adulthood. J Clin Endocrinol Metab 2014;99:1189_ 1195.

-2 Leunissen RW, Kerkhof GF, Stijnen T, Hokken-Koelega A: Timing and tempo of firstyear rapid growth in relation to cardiovascular and metabolic risk profile in early adulthood. JAMA 2009;301:2234-2242.

$>3$ Singhal A, Lucas A: Early origins of cardiovascular disease: is there a unifying hypothesis? Lancet 2004;363:1642-1645.

4 Kerkhof GF, Hokken-Koelega AC: Rate of neonatal weight gain and effects on adult metabolic health. Nat Rev Endocrinol 2012;8: 689-692.

5 Shamir R: The Benefits of Breast Feeding. Nestle Nutr Inst Workshop Ser 2016;86:6776.

-6 Anderson AK: Association between infant feeding and early postpartum infant body composition: a pilot prospective study. Int J Pediatr 2009;2009:648091.

7 De Curtis M, Pieltain C, Studzinski F, $>17$ Moureau V, Gerard P, Rigo J: Evaluation of weight gain composition during the first 2 months of life in breast- and formula-fed term infants using dual energy X-ray absorptiometry. Eur J Pediatr 2001;160:319-320.

$>8$ Fields DA, Gilchrist JM, Catalano PM, Gianni ML, Roggero PM, Mosca F: Longitudinal body composition data in exclusively breastfed infants: a multicenter study. Obesity (Silver Spring) 2011;19:1887-1891.

$>9$ Gianni ML, Roggero P, Orsi A, Piemontese P, Garbarino F, Bracco B, Garavaglia E, Agosti $\mathrm{M}$, Mosca F: Body composition changes in the first 6 months of life according to method of feeding. J Hum Lact 2014;30:148-155.

$>10$ Au CP, Raynes-Greenow CH, Turner RM, Carberry AE, Jeffery H: Fetal and maternal factors associated with neonatal adiposity as measured by air displacement plethysmography: a large cross-sectional study. Early Hum Dev 2013;89:839-843.

$\checkmark 11$ Hull HR, Dinger MK, Knehans AW, Thompson DM, Fields DA: Impact of maternal body mass index on neonate birthweight and body composition. Am J Obstet Gynecol 2008;198: 416.e411-e416.

12 Centraal Bureau voor de Statistiek: Lengte en gewicht van personen, ondergewicht en overgewicht; vanaf 1981. Centraal Bureau voor de Statistiek, 2012.
13 Breij LM, Kerkhof GF, De Lucia Rolfe E, Ong KK, Abrahamse-Berkeveld M, Acton D, Hokken-Koelega ACS: Longitudinal fat mass and visceral fat during the first 6 months after birth in healthy infants: support for a critical window for adiposity in early life. Pediatr Obes 2017;12:286-294.

14 Ellis KJ, Yao M, Shypailo RJ, Urlando A, Wong WW, Heird WC: Body-composition assessment in infancy: air-displacement plethysmography compared with a reference 4-compartment model. Am J Clin Nutr 2007; 85:90-95.

5 Ma G, Yao M, Liu Y, Lin A, Zou H, Urlando A, Wong WW, Nommsen-Rivers L, Dewey KG: Validation of a new pediatric air-displacement plethysmograph for assessing body composition in infants. Am J Clin Nutr 2004;79:653-660.

$>16$ Ferreira AP, da Silva Junior JR, Figueiroa JN, Alves JG: Abdominal subcutaneous and visceral fat thickness in newborns: correlation with anthropometric and metabolic profile. J Perinatol 2014;34:932-935.

17 Goran MI, Gower BA: Relation between visceral fat and disease risk in children and adolescents. Am J Clin Nutr 1999;70:149S-156S.

18 De Lucia Rolfe E, Modi N, Uthaya S, Hughes IA, Dunger DB, Acerini C, Stolk RP, Ong KK: Ultrasound estimates of visceral and subcutaneous-abdominal adipose tissues in infancy. J Obes 2013;2013:951954.

19 Institute of $\mathrm{M}$ : National Research Council Committee to Reexamine IOMPWG, 2009.

20 Standaard Onderwijs Indeling, Centraal Bureau van de Statistiek, 2006.

21 Schonbeck Y, Talma H, van Dommelen P, Bakker B, Buitendijk SE, Hirasing RA, van Buuren S: Increase in prevalence of overweight in Dutch children and adolescents: a comparison of nationwide growth studies in 1980, 1997 and 2009. PLoS One 2011;6: e27608.

22 Sainz RD, Urlando A: Evaluation of a new pediatric air-displacement plethysmograph for body-composition assessment by means of chemical analysis of bovine tissue phantoms. Am J Clin Nutr 2003;77:364-370.

23 Urlando A, Dempster P, Aitkens S: A new air displacement plethysmograph for the measurement of body composition in infants. Pediatr Res 2003;53:486-492.

24 Ay L, Hokken-Koelega AC, Mook-Kanamori DO, Hofman A, Moll HA, Mackenbach JP, Witteman JC, Steegers EA, Jaddoe VW:
Tracking and determinants of subcutaneous fat mass in early childhood: the Generation $\mathrm{R}$ Study. Int J Obes (Lond) 2008;32:1050-1059.

25 Gesta S, Tseng YH, Kahn CR: Developmental origin of fat: tracking obesity to its source. Cell 2007;131:242-256.

-26 Gishti O, Gaillard R, Durmus B, Abrahamse $M$, van der Beek EM, Hofman A, Franco OH, de Jonge LL, Jaddoe VW: BMI, total and abdominal fat distribution, and cardiovascular risk factors in school-age children. Pediatr Res 2015;77:710-718.

27 Liu KH, Chan YL, Chan WB, Kong WL, Kong MO, Chan JC: Sonographic measurement of mesenteric fat thickness is a good correlate with cardiovascular risk factors: comparison with subcutaneous and preperitoneal fat thickness, magnetic resonance imaging and anthropometric indexes. Int J Obes Relat Metab Disord 2003;27:1267-1273.

28 Kerkhof GF, Leunissen RW, Hokken-Koelega AC: Early origins of the metabolic syndrome: role of small size at birth, early postnatal weight gain, and adult IGF-I. J Clin Endocrinol Metab 2012;97:2637-2643.

29 Eriksson JG: Early growth and coronary heart disease and type 2 diabetes: findings from the Helsinki Birth Cohort Study (HBCS). Am J Clin Nutr 2011;94:1799S-1802S.

30 Liem ET, De Lucia Rolfe E, L'Abee C, Sauer PJ, Ong KK, Stolk RP: Measuring abdominal adiposity in 6 to 7 -year-old children. Eur J Clin Nutr 2009;63:835-841.

-31 Armstrong J, Reilly JJ, Child Health Information T: Breastfeeding and lowering the risk of childhood obesity. Lancet 2002;359:20032004.

32 Booth A, Magnuson A, Foster M: Detrimental and protective fat: body fat distribution and its relation to metabolic disease. Horm Mol Biol Clin Invest 2014;17:13-27.

-33 Breij LM, Steegers-Theunissen RP, Briceno D, Hokken-Koelega AC: Maternal and fetal determinants of neonatal body composition. Horm Res Paediatr 2015;84:388-395.

34 Josefson JL, Hoffmann JA, Metzger BE: Excessive weight gain in women with a normal pre-pregnancy BMI is associated with increased neonatal adiposity. Pediatr Obes 2013;8:e33-e36.

-35 Ay L, Van Houten VA, Steegers EA, Hofman A, Witteman JC, Jaddoe VW, Hokken-Koelega AC: Fetal and postnatal growth and body composition at 6 months of age. J Clin Endocrinol Metab 2009;94:2023-2030. 